\title{
Title: Prematurity, the Diagnosis of Bronchopulmonary Dysplasia, and Maturation of Ventilatory Control Abbreviated Title: Control of Breathing and the Diagnosis of BPD
}

\author{
Daniel Mammel ${ }^{1}$ and James Kemp ${ }^{1}$ \\ ${ }^{1}$ Washington University School of Medicine in Saint Louis
}

December 2, 2020

\begin{abstract}
Infants born before 32 weeks post-menstrual age (PMA) and receiving respiratory support at 36 weeks PMA are diagnosed with bronchopulmonary dysplasia. This label suggests that their need for supplemental oxygen is primarily due to acquired dysplasia of airways and airspaces, and that the supplemental oxygen $(\mathrm{O} 2)$ is treating residual parenchymal lung disease. However, current approaches to ventilatory support in the first days of life, including artificial surfactant use and lower ventilating pressures have changed the pathology of chronic lung disease, and emerging evidence suggests that immature ventilatory control may also contribute to the need for supplemental oxygen at 36 weeks PMA. In all newborns, maturation of ventilatory control continues ex utero and is a plastic process. Supplemental O2 mitigates the hypoxemic effects of delayed maturation of ventilatory control, as well as reduces the duration and frequency of periodic breathing events. Prematurity is associated with altered and occasionally aberrant maturation of ventilatory control. Infants born prematurely, with or without a diagnosis of BPD, are more prone to long-lasting effects of dysfunctional ventilatory control. Awareness of the interaction between parenchymal lung disease and delayed maturation of ventilatory control is essential to understanding why a given premature infant requires and is benefitting from supplemental $\mathrm{O} 2$ at 36 weeks PMA.
\end{abstract}

Title : Prematurity, the Diagnosis of Bronchopulmonary Dysplasia, and Maturation of Ventilatory Control Authors : Daniel M. Mammel MDa, James S. Kemp MD

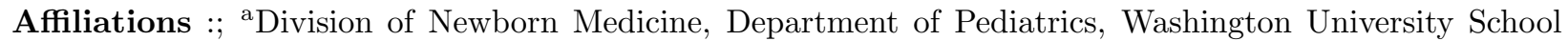
of Medicine, St. Louis, MO. ${ }^{\mathrm{b}}$ Division of Allergy, Immunology and Pulmonary Medicine, Department of Pediatrics, Washington University School of Medicine, St. Louis, MO

\section{Corresponding Author :}

James S. Kemp MD

Washington University, Department of Pediatrics

Campus Box 8116

660 S. Euclid Avenue

St. louis, MO 63110

Phone: 314-747-5173

Fax: 314-454-2515 
Funding : Physiologic Biomarkers Predicting Ventilatory Instability and Hypoxemia in Premature Infants (PreVent) NHLBI, U01 HL133700-01

Keywords : BPD; sleep disordered breathing; periodic breathing; sudden unexpected infant death; SUID; chronic lung disease; CLD

Abbreviated Title : Control of Breathing and the Diagnosis of BPD

\section{Hosted file}

CoB Review 12.1 final.pdf available at https://authorea.com/users/380708/articles/496580title-prematurity-the-diagnosis-of-bronchopulmonary-dysplasia-and-maturation-ofventilatory-control-abbreviated-title-control-of-breathing-and-the-diagnosis-of-bpd
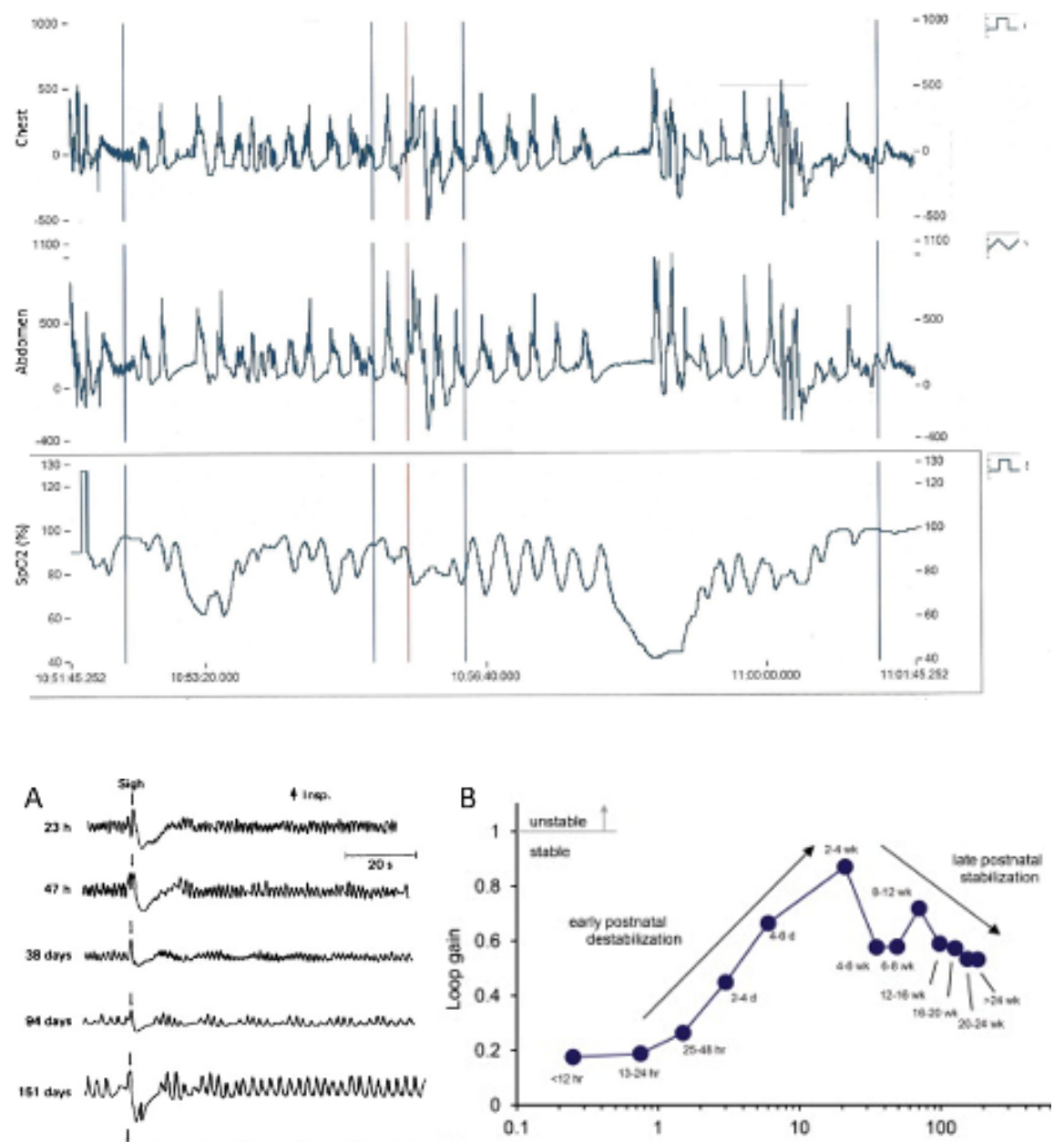


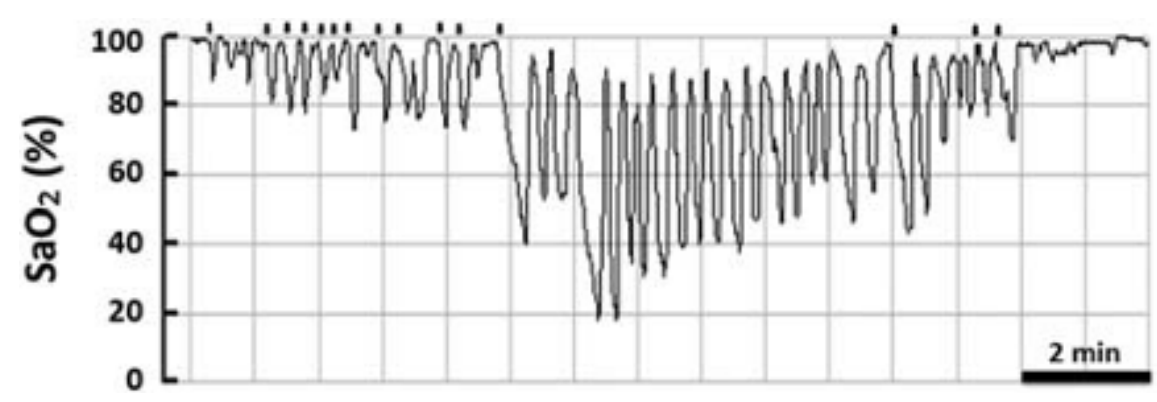

Factors that contribute to Loop Gain

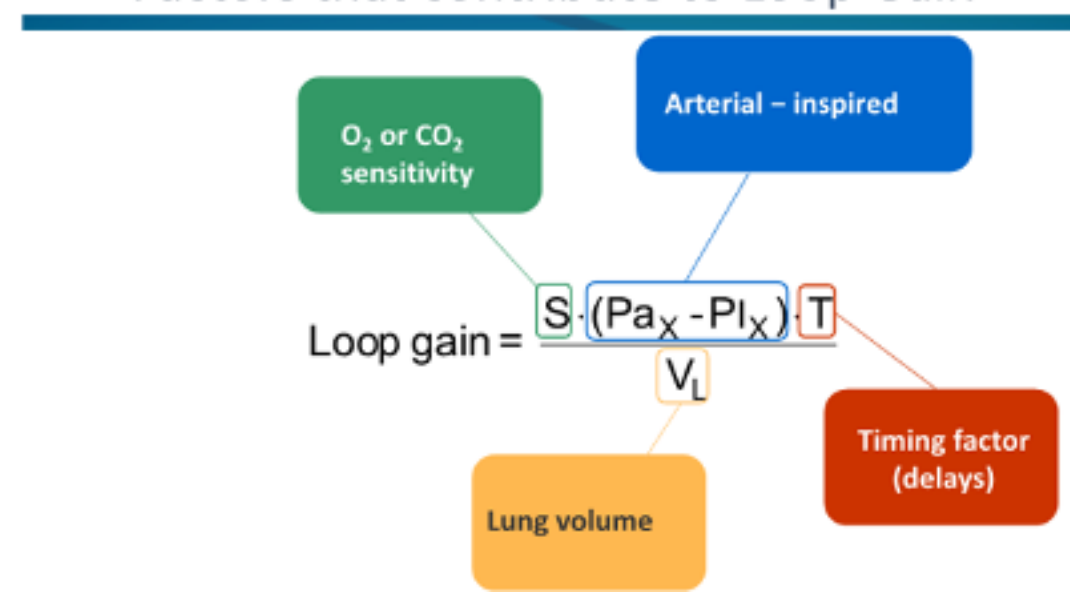



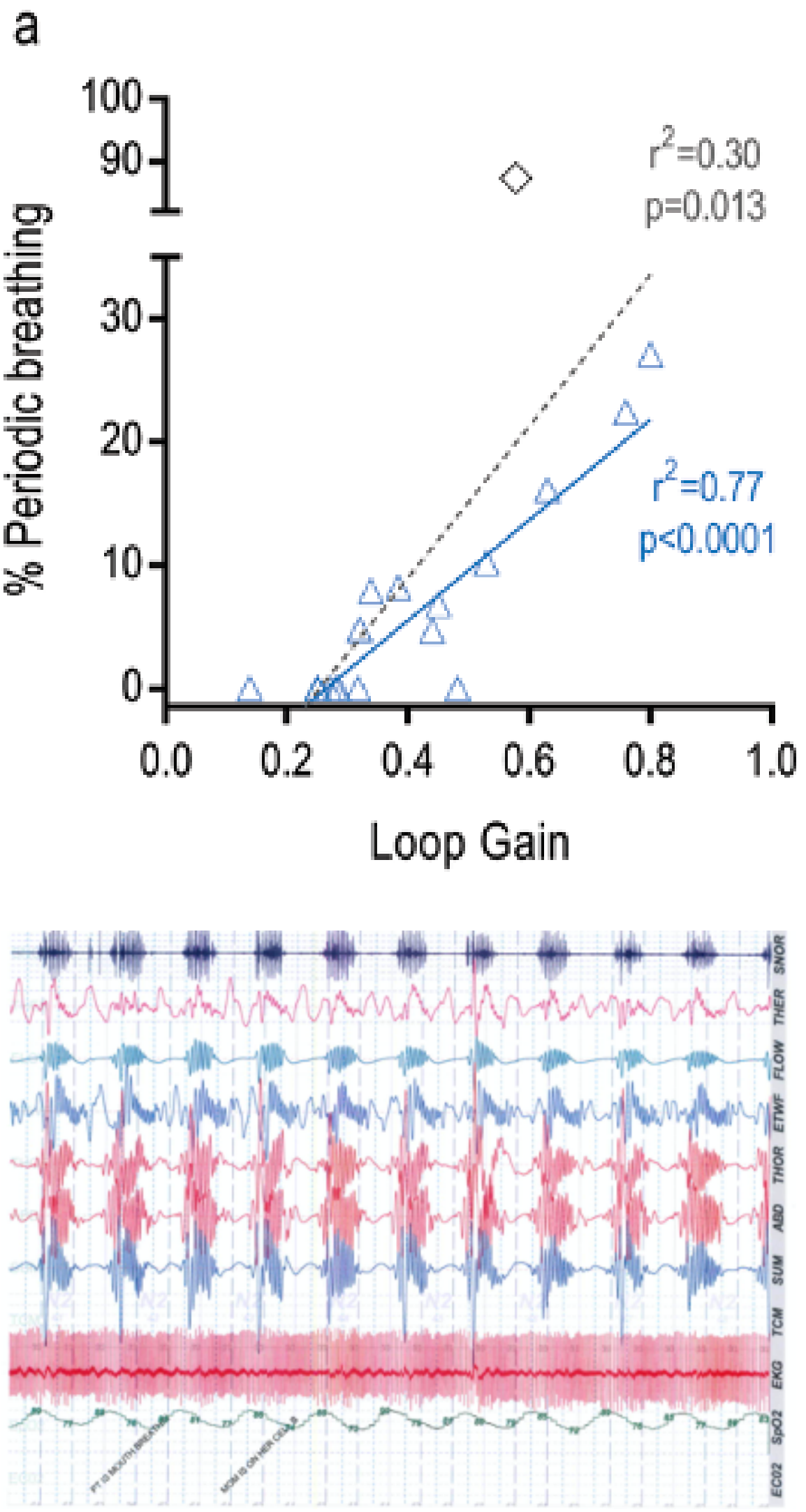


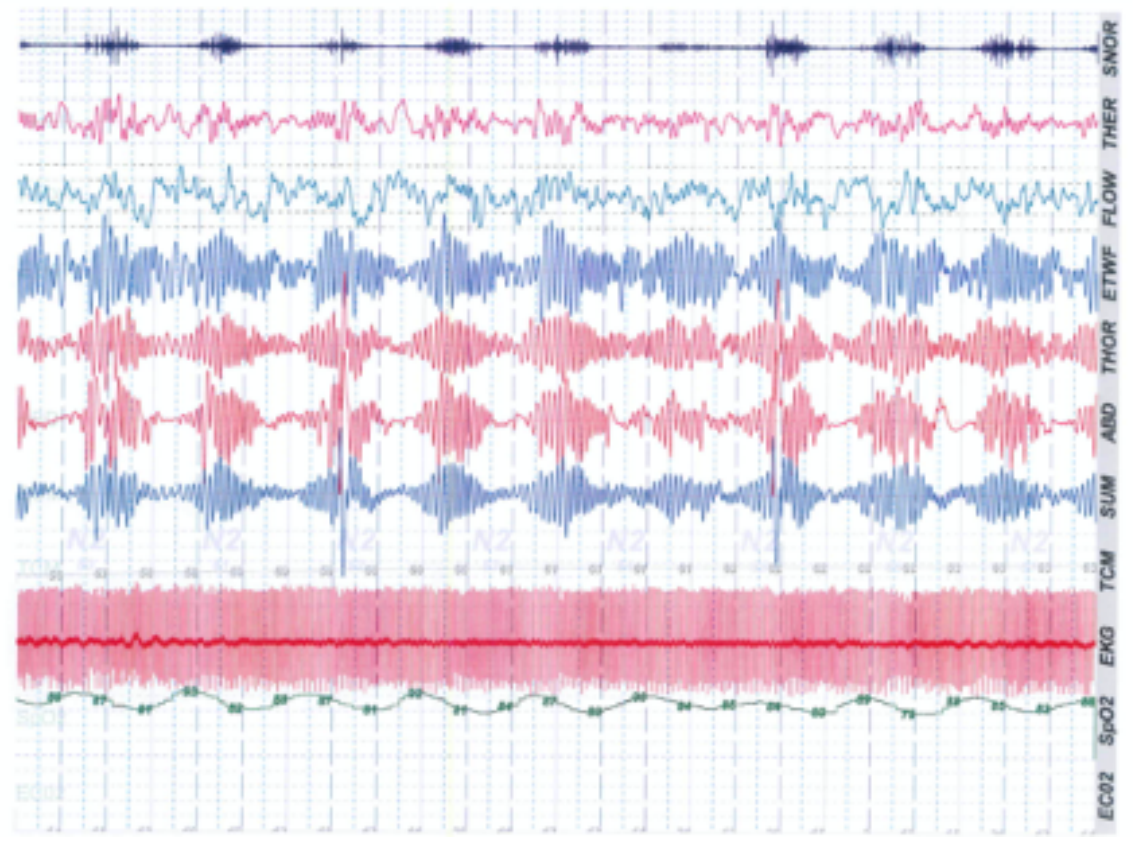

